\title{
Lean and BIM meet social sciences: new perspectives in construction engineering and management
}

\author{
Special Editors \\ Carlos T. Formoso Federal University of Rio Grande do Sul, Brazil \\ Patricia Tzortzopoulos University of Huddersfield, U.K. \\ Daniel Forgues École de Technologie Supérieure, Canada
}

